Methods We recruited 25 MSM (mean age $=35 ; \mathrm{SD}=11 ; 88 \%$ Black) following their participation in Safe Spaces and Places (SSP) - a community-based, online-linked, mobile van HIV/ STI testing modality in Baltimore, MD. Participants completed 60-minute semi-structured interviews exploring their perceptions of SSP relative to clinic-based testing experiences. Interview transcripts were doubled coded until there was group consensus and analyzed using a constant comparative approach.

Results Participants described a preference for the SSP testing modality compared to their clinic-based testing experiences. Three themes related to this preference emerged from the interviews: 1) greater comfort with staff and testing environment - e.g. 'it was like a different environment than being in a hospital. And everybody was real kind and polite;' 2) less concern with maintaining privacy/confidentiality - e.g. 'I felt like the van is much more discrete and private... When you're in the [STD clinic], you're out there with a bunch of people-

...it's just really crowded;' and 3) increased accessibility - e.g. 'I did really like the van being in the evening hours, being offered multiple days per week so that I could fit it in my schedule. I like that it shows up in different places in the community.'

Discussion Our findings suggest this majority Black sample of MSM preferred the SSP testing modality because it removed barriers to HIV/STI testing for this priority population. Increasing access to HIV/STI testing in BMSM will require identifying barriers to testing in this population and developing modalities that attend to these barriers.

\section{P400 FACTORS ASSOCIATED WITH PRE-EXPOSURE PROPHYLAXIS USAGE AMONG MEN WHO HAVE SEX WITH MEN IN BALTIMORE, MARYLAND}

${ }^{1} \mathrm{~N}$ Thornton*, 'J Jennings, 'J Perin, 'S Huettner, ${ }^{2} \mathrm{D}$ German, ${ }^{1} \mathrm{E}$ Fields. 'Johns Hopkins School of Medicine, Baltimore, USA; ${ }^{2}$ Johns Hopkins Bloomberg School of Public Health, Baltimore, USA

\subsection{6/sextrans-2021-sti.430}

Background Men who have sex with men (MSM), particularly Black and adolescent/younger adult (AYA) MSM, are disproportionally impacted by the US HIV epidemic. PrEP may mitigate disparities, but uptake remains low among these populations. This study examines factors associated with PrEP usage in a majority Black and AYA MSM population.

Methods Data from two behavioral surveillance studies in Baltimore, Maryland, BESURE (2017, venue-based time-location sampling) and Safe Spaces and Places (2018, online-based time-location sampling), was used to determine factors associated with PrEP usage among HIV-negative MSM reporting previous knowledge of PrEP, using bivariate and multivariable logistic regressions.

Results $302 \mathrm{HIV}$-negative MSM were included in the analysis. The mean age was $33.1(\mathrm{SD}=9.64$; range $=19-68)$. The majority $(67.6 \%)$ were AYA (18-34 years-old) and self-identified as Black (69.9\%). Health care access was high with $17.9 \%$ reporting inability to afford needed care. $45.5 \%$ reported experiencing discrimination related to their sexuality. Using the CDC MSM risk index, a significantly lower percentage of Black vs. non-Black (38.4\% vs. $53.9 \%$; p-value $=0.01)$, and higher percentage of AYA vs. older $(47.6 \%$ vs. $33.7 \%$; pvalue $=0.02$ ) participants were PrEP indicated based on self- reported risk behaviors. 20.2\% of all participants reported prior year PrEP usage with no significant differences by race or age. In bivariate analyses, higher education, PrEP indication, and experiencing discrimination were associated with increased odds of usage. In the multivariable model, only PrEP indication and higher education remained associated.

Conclusion In this majority AYA and Black MSM sample, PrEP usage remains low despite increased HIV incidence compared to other populations. PrEP indication was associated with increased usage; however, PrEP indication was significantly lower among Black MSM. This suggests that the risk index may underestimate HIV acquisition risk among Black MSM and may be necessary but insufficient as a clinical tool for determining eligibility or promoting uptake in this population.

\section{P401 DEVELOPMENT AND UTILIZATION OF ANTIBODIES SPECIFIC FOR EXTRACELLULAR LOOPS OF THE TREPONEMA PALLIDUM OUTER MEMBRANE PROTEIN BAMA (TP0326)}

${ }^{1} \mathrm{~S}$ McBride, ${ }^{1} \mathrm{M}$ Ferguson, ${ }^{1} \mathrm{M}$ Kelly, ${ }^{2,3} \mathrm{~K}$ Hawley, ${ }^{3} \mathrm{~A}$ Luthra, ${ }^{1} \mathrm{H}$ Driscoll, ${ }^{3} \mathrm{~J}$ MontezumaRusca, ${ }^{3} \mathrm{~K}$ Delgado, ${ }^{3} \mathrm{M}$ Caimano, ${ }^{3} \mathrm{~J}$ Radolf, ${ }^{4} \mathrm{M}$ Moody, ${ }^{5} \mathrm{M}$ Weiner ${ }^{*}$. ${ }^{1} \mathrm{Abcam}$, Branford, USA; ${ }^{2}$ Connecticut Children's, USA; ${ }^{3}$ UConn Health, Farmington, USA; ${ }^{4}$ Duke Human Vaccine Institute, Durham, USA; ${ }^{5}$ AbbraTech, Gilford, USA

\subsection{6/sextrans-2021-sti.431}

Background Researchers often generate antibodies (Abs) to epitopes on the surface of a protein or in a hydrophilic loop. It can be more difficult to isolate phage displayed antibodies (Abs) that bind preferentially at or near a specific amino acid on a protein or peptide. We used a phage-display method called 'DPAC' to test the hypothesis that opsonic antibodies can be generated against extracellular loops (ECLs) of Treponema pallidum ( $\mathrm{Tp}$ ) outer membrane proteins (OMPs). As proof of concept, we focused on BamA-ECL4 which we previously showed is a target for opsonic antibodies.

Methods A modified peptide incorporating a non-native amino acid (nnAA) at a desired site was designed for BamA-ECL4. Antibody phage display in vitro selection was used to isolate nnAA-specific and nnAA-preferred $\mathrm{scFv}$ binding phage. AXM Mutagenesis was then used for directed evolution to alter the specificity of several discovered nnAA-binding phage to (i) recognize the same peptide, but with a native amino acid at the nnAA site and (ii) have a lower binding affinity. Specific clones were converted from $\mathrm{scFv}$ to IgGs, expressed and then purified and retested for specificity and affinity. We validated our antibodies using a Pyrococcus furiosus thioredoxin (PfTrx) as a scaffold to present BamA ECL4.

Results We successfully used the in vitro approach to derive site-specific anti-BamA ECL4 Abs and evolved non-synonymous native-specific Abs to significantly improve affinity. These antibodies bound PfTrx-BamA-ECL4 and did not bind control peptides, indicating that these Abs were ECL4-specific. Testing of these antibodies in a mouse opsonophagocytosis assay is underway.

Conclusion When used in conjunction with OMP structural models, DPAC and PfTrx scaffolds are promising tools for identifying and characterizing antigenic ECLs. Identification of anti-ECL monoclonal antibodies with opsonic activity will provide evidence to support the selection of specific T. pallidum OMPs for vaccine development. 